\title{
Influence of the Framework Material on the Biomechanical Behaviour of Dental Restorations
}

Ludger Keilig ${ }^{1,2}$, Helmut Stark², Christoph Bourauel ${ }^{1}$

1 Oral Technology and ${ }^{2}$ Department of Prosthetic Dentistry, Preclinical Education and Materials Science, Dental School, University of Bonn, Germany

\section{Abstract}

The choice of different framework materials (like ceramics, precious or non-precious alloys, and high performance polymers) influence the biomechanical behaviour of implant-supported prosthetic restorations. This influence changes with the implant's state of osseointegration. It is the
influences using numerical methods.

3D models of a four unit bridge on two implants as well as a full arch restoration on four implants were created. Following framework materials were simulated: Gold alloy, immediate loading as well as after osseointegration.

Full osseointegration decreased the calculated displacements of the framework compared to immediate loading for the same model, and resulted in a noticeable Loading single implants under immediate loading condition resulted in excessive strains up to tenfold the typically assumed non-pathological load After osseointegration the strains in the bone were reduced below the pathological limits for all materials.

The simulations showed a large influence of the state of osseointegration on the resulting stresses and strains in the involved biological tissues. After osseointegration strains in the surrounding biological tissues.

\section{Background and Aim}

Framework materials for implant-supported restorations, such as ceramics, precious or non-precious alloys, differ widely in their material properties, especially with respect to the mechanical behaviour. The range of these material properties is increased with the introduction of alternative framework materials like high-performance polymers. Especially for immediate and early loading protocols the load transfer from the restoration to the implants may influence the ongoing from the restoratic
osseointegration.

It was the aim of this study to determine the biomechanical behaviour of different implant-supported restorations depending on the framework material using numerical methods (finite element methods, FEM), and material using numerical methods (finite element methods, FEM), and
compare this behaviour under immediate loading as well as after osseointegration.

\section{Methods and Materials}

FE models of two different implant-supported restorations were generated based on clinical data, first a four unit (4U) bridge on two implants, second a full arch restoration on four implants. Each model included cortical as well as spongious bone, mucosa, implants, abutments, framework and veneering. Following framework materials were simulated: Gold alloy, Titanium, Pekkton as an high performance polymer (see Table 1 for the material parameters used).

Different loading situations with forces up to $400 \mathrm{~N}$ were simulated: load application above the central unit of the bridges, above single implants, of evenly distributed on all implants. A first simulation series defined a sliding contact between implants and bone to simulate the immediate loading condition; a second series used a fixed connection between implants and bone to simulate the osseointegrated state. All oading situations were analysed with immediate loading as well as after osseointegration.
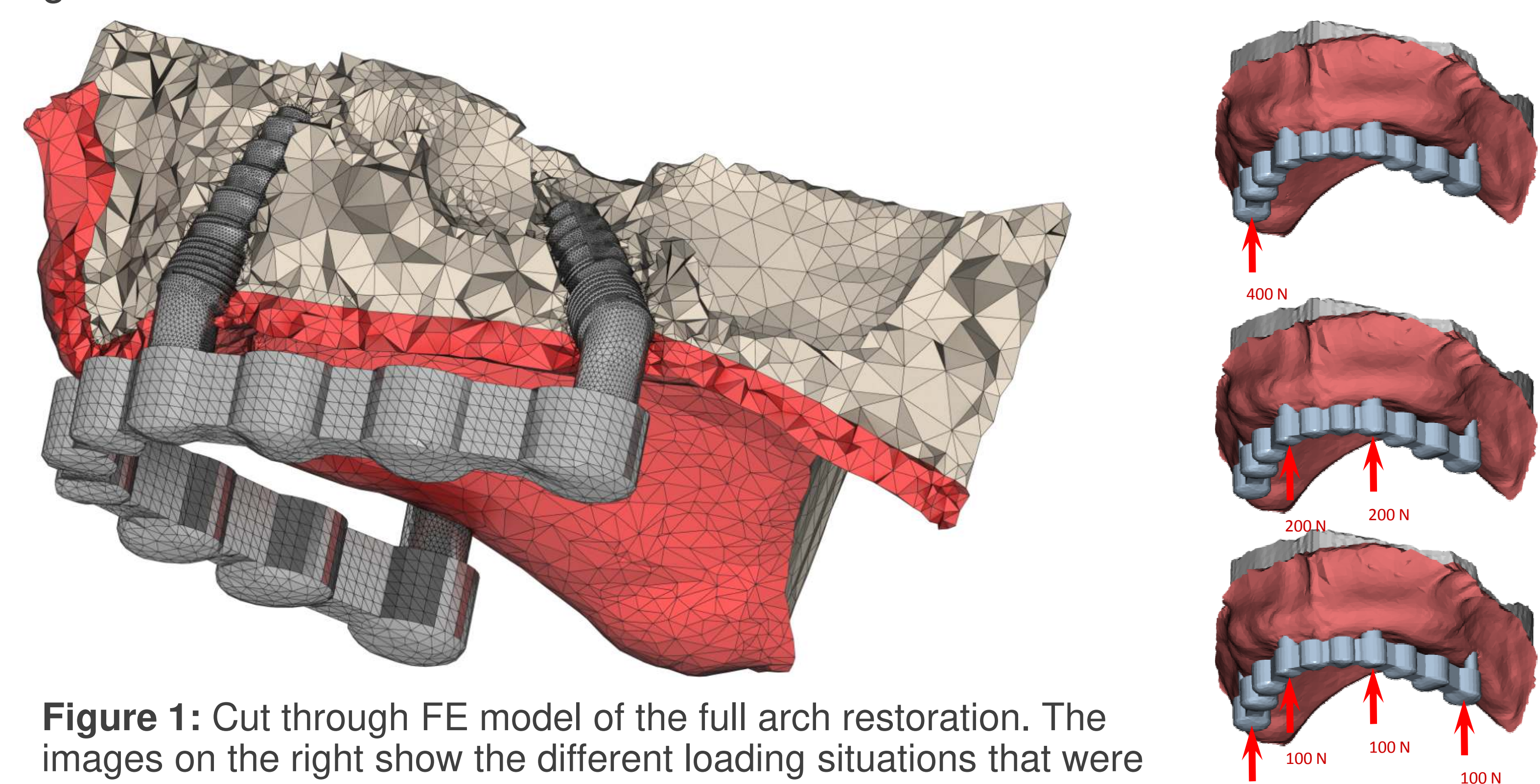

Figure 1: Cut through FE model of the full arch restoration. The considered in this model.

\begin{tabular}{lrc}
\hline \multicolumn{1}{c}{ Material } & $\begin{array}{r}\text { Young's } \\
\text { Modulus }\end{array}$ & $\begin{array}{c}\text { Poisson' } \\
\text { s Ratio }\end{array}$ \\
\hline Tooth & $20 \mathrm{GPa}$ & 0.30 \\
Titanium (implant/framework) & $110 \mathrm{GPa}$ & 0.30 \\
Cortical bone & $18 \mathrm{GPa}$ & 0.30 \\
Spongious bone & $0.3 \mathrm{GPa}$ & 0.30 \\
Cement & $3 \mathrm{GPa}$ & 0.30 \\
Veneering & $6.6 \mathrm{GPa}$ & 0.25 \\
Gold alloy (framework) & $136 \mathrm{GPa}$ & 0.30 \\
Pekkton (high performance & $4.5 \mathrm{GPa}$ & 0.25 \\
polymer) & & \\
\hline
\end{tabular}

Table 1: List of material parameters used in the

simulations.

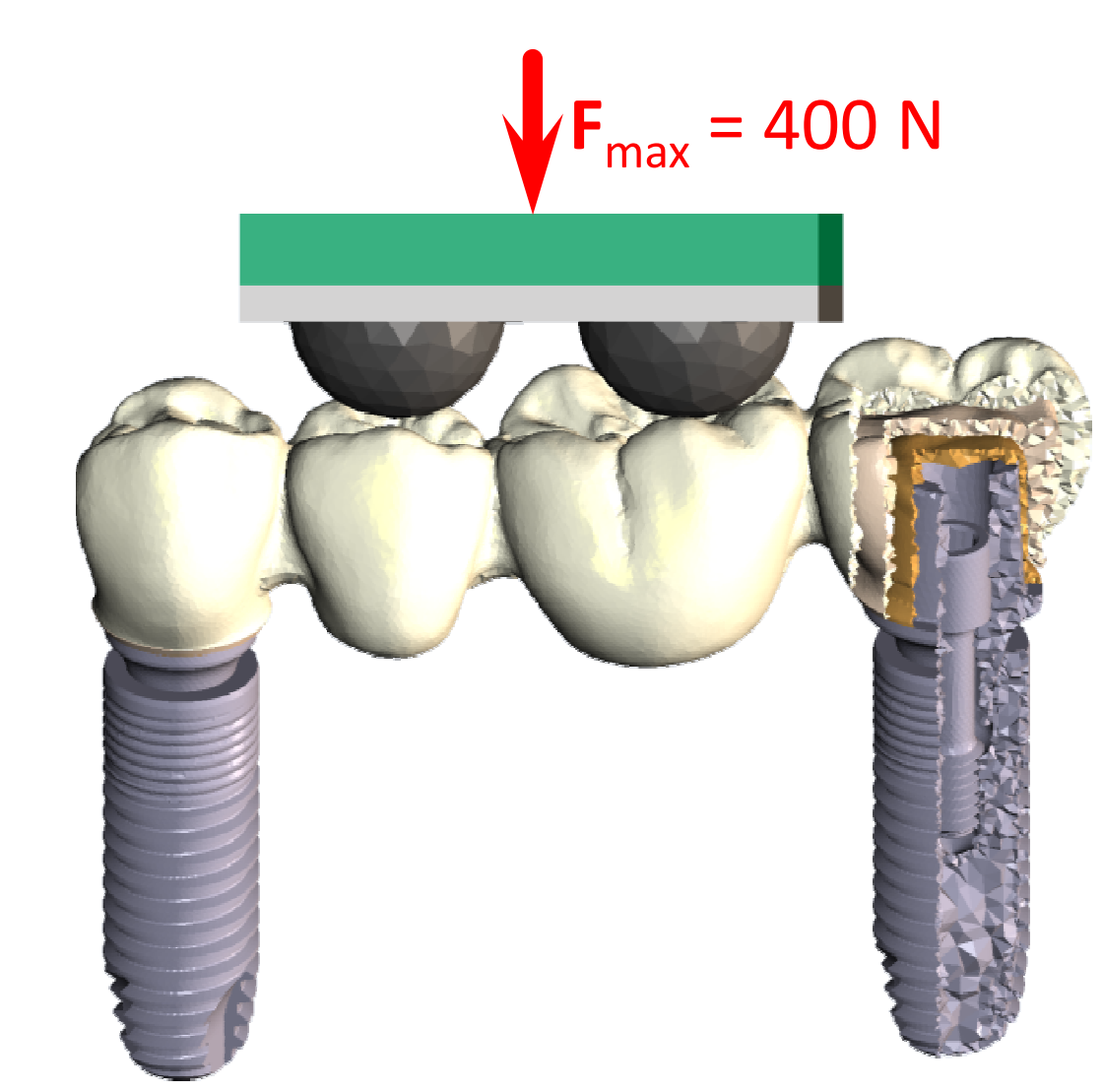

Figure 2: FE model of $4 \mathrm{U}$

\section{Results}

The biomechanical reaction of the framework upon loading varied depending on the framework material and the state of the osseointegration of the implants. Figure 3 shows the displacement for
some of the simulations with the full arch restoration, while Figure 4 shows the strains in the bone for the same models.

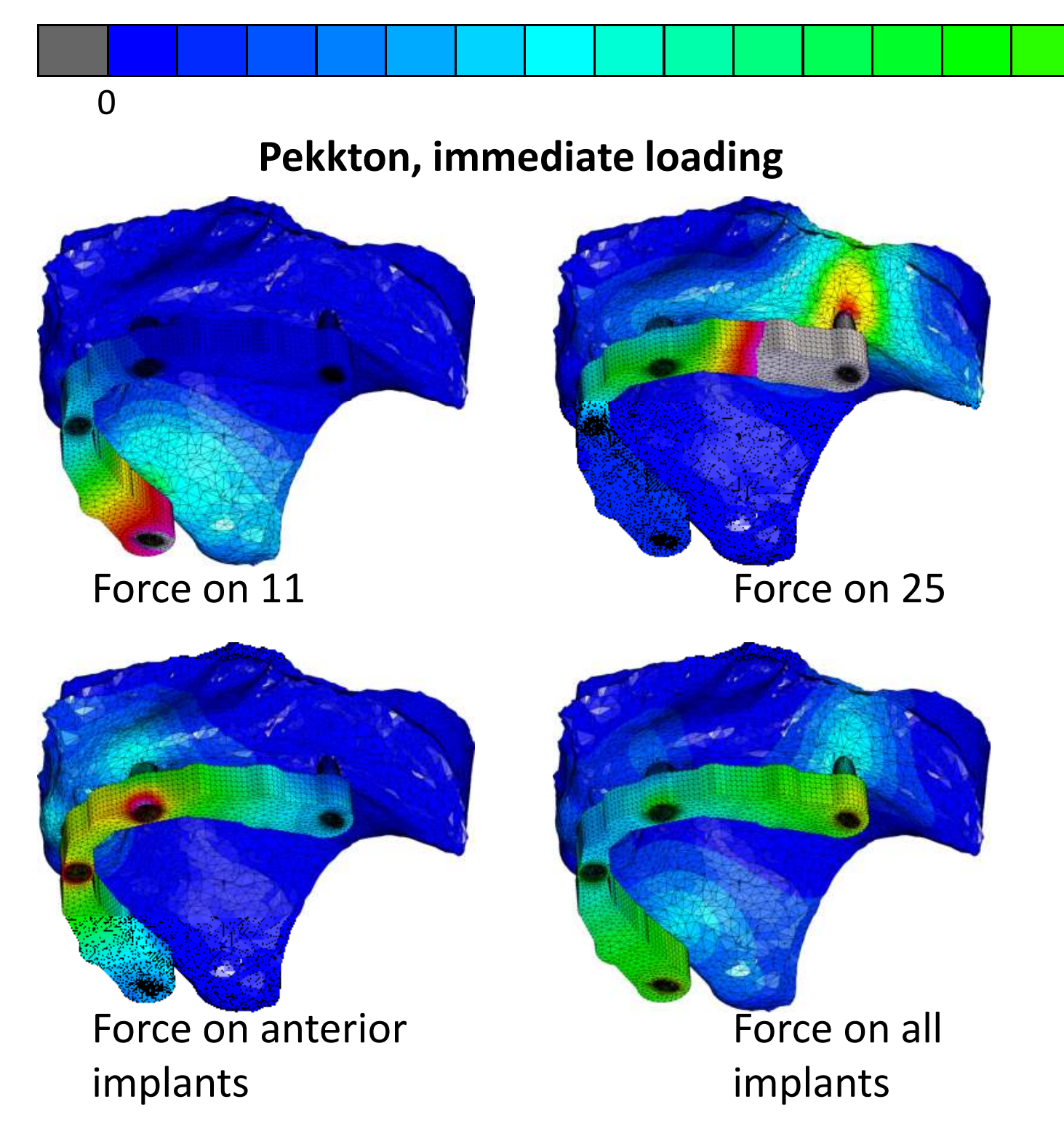

Figure 3: Calculated displacements in the models of a full-arch restoration. The area force application is denoted below each image.

Immediate loading of the framework in the region of an implant resulted in displacements of up to $50 \mu \mathrm{m}$ for the polymer fram work and up to $37 \mu \mathrm{m}$ for the Titanium famework. Assuming full osseointegration the framework by approximately $10 \%$ compared to immediate loading for the same model depending on the area of force application.
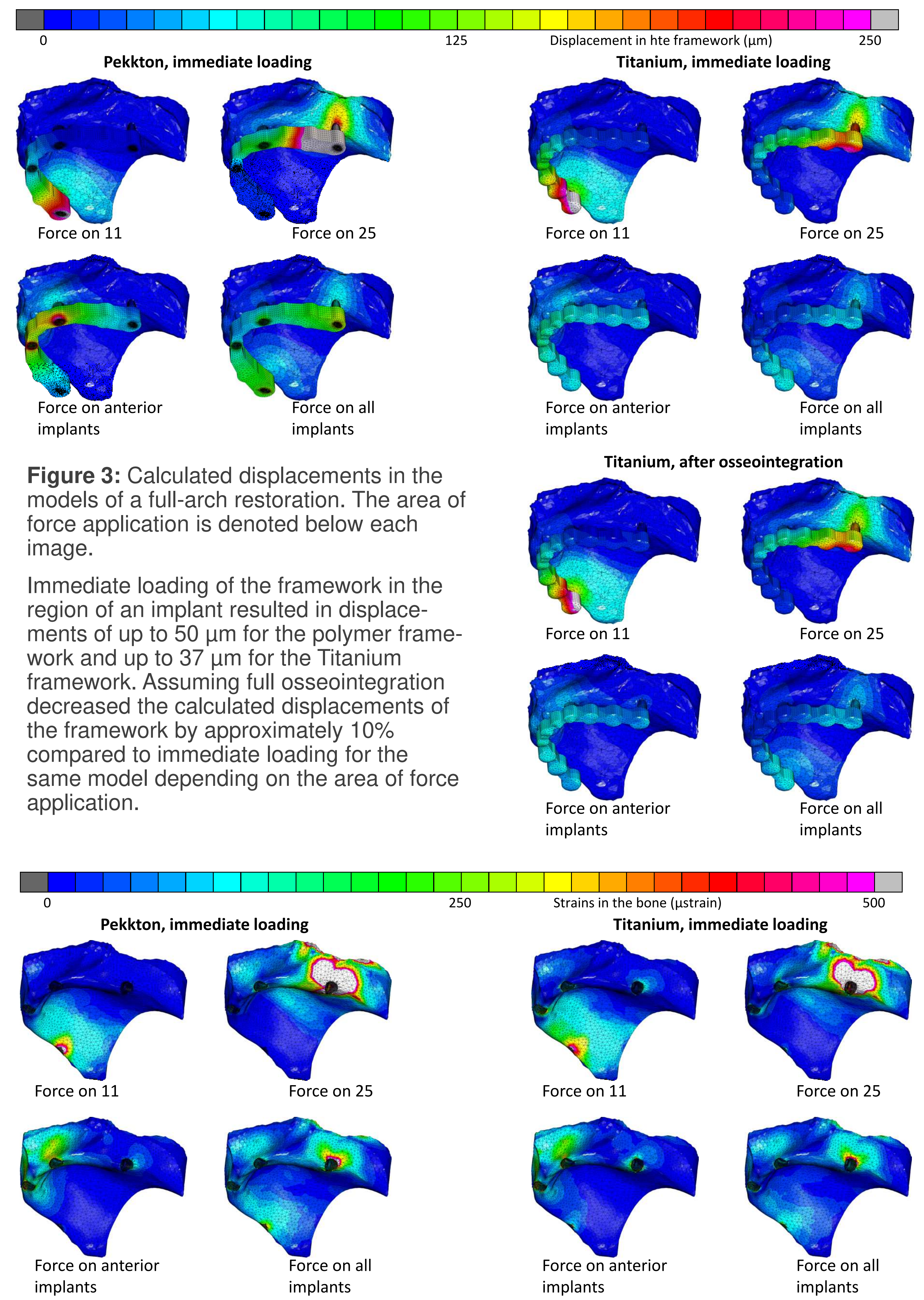

Figure 4: Calculated strains in the model of full-arch restoration.

On immediate loading, high peak values were found for the calculated strains, especially in the bone region at the implant threads. Loading single implants under immediate loading condition resulted in local areas of excessive strains of above $48,000 \mu$ strain, which is tenfold the typically assumed non4.000 strains [1]). After osseointegration the strains in the bone were reduced below the pathological limits for all materials. Especially for the osseointegrated situation the framework material did not influence magnitude or distribution of stresses and strains in the

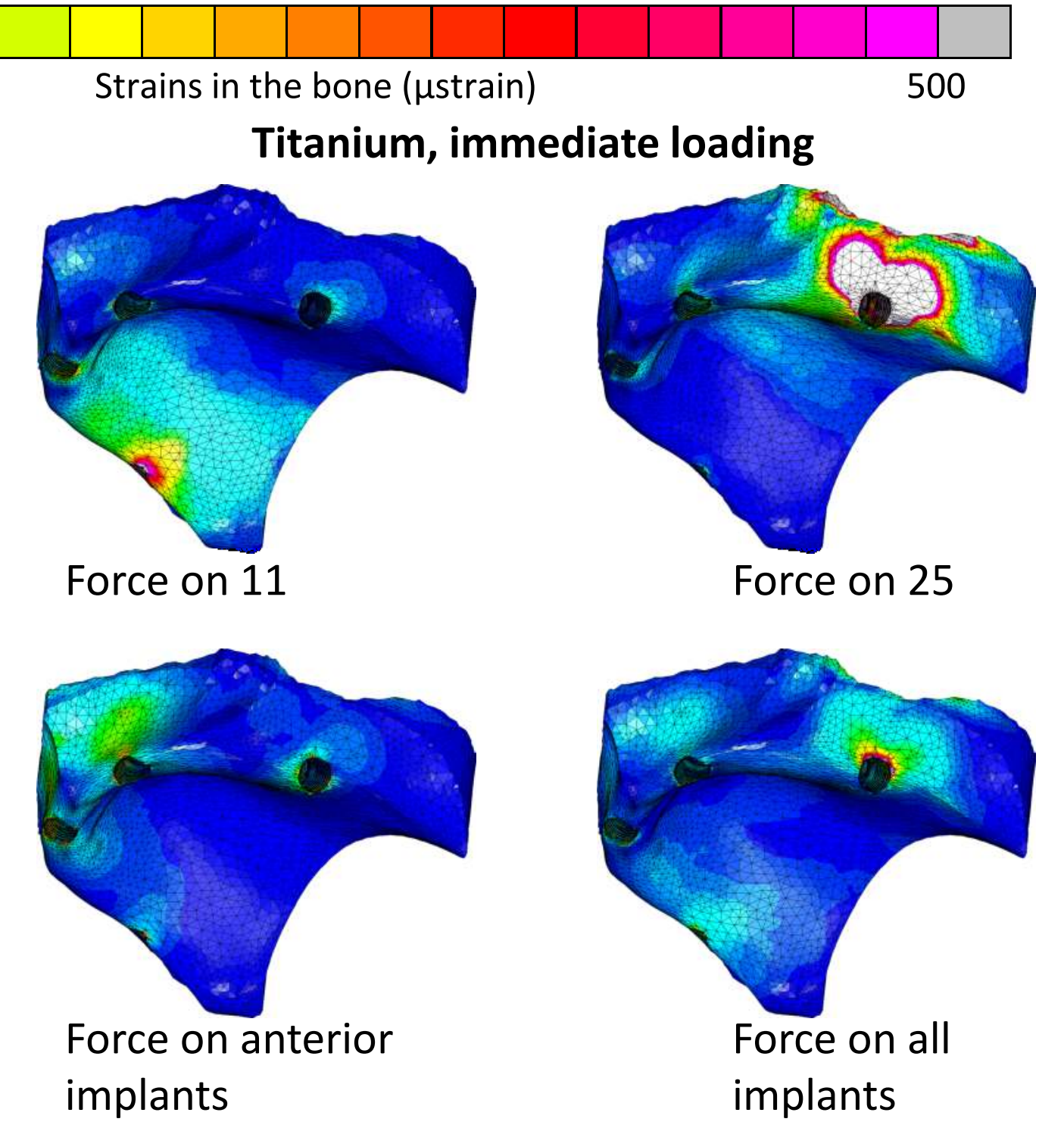
implants

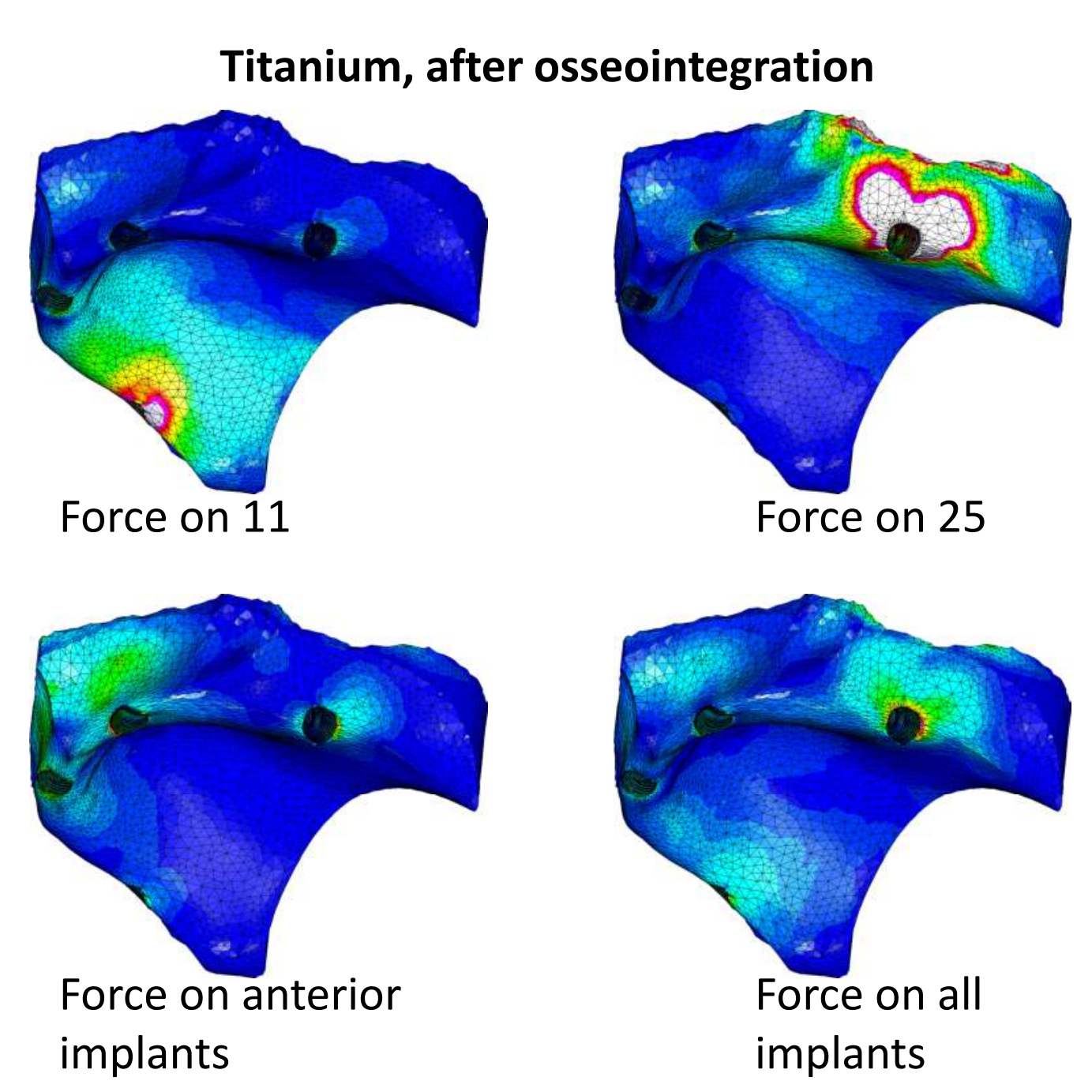
surrounding biological tissues.

\section{Conclusions}

While the load determined for the immediate loading situation within the bone is rather high, the assumed maximum load of $400 \mathrm{~N}$ is not a common load regularly observed in the patient's mouth. The performed simulations showed a large influence of the state of osseointegration on the resulting stresses and strains in the involved biological tissues. After achieving a complete osseointegration, the framework material only had a minor influence on the resulting biomechanical load within the bone.

\section{References}

Frost HM. Bone's mechanostat: A 2003 update. Anat. Rec. A Discov. Mol. Cell. Evol. Biol. 2003; 275A // 275(2):1081-101. 